\title{
Modelos de colaboración para la atención a la salud mental en Andalucía: evaluación tras 9 años de implantación.
}

\author{
Collaborative models for mental health care in Andalusia: an assessment after 9 years of \\ implementation.
}

\author{
José Manuel Aranda Regules a, Asunción González Villalba ${ }^{\text {b }}$, Vanessa Ropero Gallardo ${ }^{\mathrm{c}}$, Mi- \\ nervina Montero Quevedo d, Alfonso Galán Pajuelo ${ }^{\mathrm{e}}$, Domingo Díaz del Peral ${ }^{\mathrm{f}}$, Rafael del Pino
}

López ${ }^{g}$.

\begin{abstract}
${ }^{a}$ Médico de familia. Centro de Salud San Andrés Torcal Málaga, España, ${ }^{b}$ Psicóloga. Técnico de Función Administrativa. UGC Salud Mental Hospital Virgen de la Victoria, Málaga, España, ${ }^{c}$ Enfermera. Centro de Salud San Andrés Torcal Málaga, España, 'Psicóloga clínica. USMC Puerta Blanca Málaga, España,

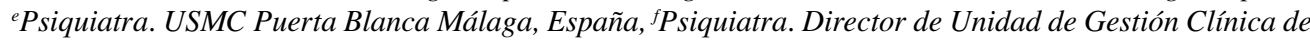
Salud Mental de Torrecárdenas. Almería, España, ${ }^{8}$ Psiquiatra. Director de Unidad de Gestión Clínica de Salud Mental Virgen de la Victoria. Málaga, España.
\end{abstract}

Correspondencia: José Manuel Aranda Regules (regules11@gmail.com)

Recibido: 10/03/2011; aceptado con modificaciones: 18/07/2011

RESUMEN: INTRODUCCIÓN: En 2002 se inició la implantación del Proceso de Atención Integrado Ansiedad, Depresión, Somatizaciones (PAIADS) en Andalucía (modelo de colaboración asistencial). Se presenta su evaluación después de 9 años.

MATERIAL Y METODOS: Análisis cualitativo de un CS urbano ${ }^{1}$ (entrevistas en profundidad, análisis documental y de registros) que implantó el PAIADS en 2002. Cuestionario telefónico a 380 Centros de Salud (CS) y 76 Unidades de Salud Mental Comunitaria (USMC) (Noviembre 2008-Mayo 2009). Estadística descriptiva de frecuencias y análisis bivariante (paquete SPSS)

RESULTADOS: El análisis cualitativo del CS muestra los 4 pilares de la implantación exitosa del PAIADS: comunicación bidireccional entre instituciones y profesionales, organización de consulta conjunta, función de filtro e inclusión de objetivos de salud mental en contrato de gestión.

La tasa de respuesta a los cuestionarios fue de $68.9 \%$ en CS y $78.9 \%$ en USMC. Solamente $2.3 \%$ de CS implantaron la totalidad del PAIADS. Sin embargo el $88.2 \%$ hace una implantación parcial de alguno de sus elementos: responsable del programa, desplazamiento periódico de especialistas a CS, formación, protocolos, consulta programada de salud mental por los médicos de familia y grupos psicoeducativos en los CS. Los análisis muestran que la satisfacción profesional alta y la menor demora para primeras consultas a atención
ABSTRACT: INTRODUCTION: The implementation of the Integrated Care Process of Anxiety, Depression and Somatization (ICPADS; a collaborative care model) began in Andalusia in 2002.. Data presented correspond to an assessment after 9 years of implementation

MATERIAL AND METHODS: Telephone questionnaire were carried out to 380 Health Centers (HC) and 76 Community Mental Health Units (CMHU) from November 2008 to May 2009. This was completed with a qualitative analysis for an urban HC (in depth interviews, document analysis) which introduced the ICPADS in 2002. We used descriptive statistics of frequencies and univariate analysis (SPSS software)

RESULTS: Qualitative analysis revealed four stone corners of a successful implementation: a two-way communication between institutions and professionals, an organization of shared consultation, a filter function and an inclusion of mental health objectives in the management contract. Response rate to the questionnaire was $68.9 \%$ in $\mathrm{HCs}$ and $78.9 \%$ in the CMHUs. ICPADS was fully implanted in $2.3 \%$ of HCs. However, a partial implantation was observed in $88.2 \%$ of the units on specific aspects as the existence of a person in charge of the program, periodic visits from mental health specialists to $\mathrm{HCs}$, continued education, protocols, specific mental health consultation from family physicians and psychoeducational groups offered in the HCs. Analyses revealed that a higher job

\footnotetext{
1 Centro de Salud San Andrés-Torcal de Málaga
} 
especializada están asociadas con la implantación del PAIADS (pacientes vistos conjuntamente, formación $\mathrm{y}$ protocolos). Adicionalmente encontramos que el mayor numero de derivaciones esta asociado a menor masificación de las consultas, ausencia de oferta de grupos psicoeducativos en los CS y no desplazamiento periódico de especialistas.

CONCLUSIONES: El PAIADS tiene capacidad de contención no medicalizadora y gestión de la demanda para estas patologías en los CS y genera satisfacción profesional sin costes añadidos de implantación o mantenimiento. Es necesario realizar investigaciones complementarias sobre su impacto en la salud mental de los pacientes.

PALABRAS CLAVE: Modelos de colaboración servicios de salud mental, atención primaria de salud. satisfaction and minor delays for a first specialist visit were associated with the implementation of ICPADS (shared consultation, continued education and protocols). In addition, an increased number of referrals were associated with a less overcrowded consultation and a lack of both psychoeducational groups offer and periodic visits from mental health specialists.

CONCLUSIONS: The ICPADS showed the capacity to solve and manage mental health demands of these pathologies in the HCs without medicalize them and generates job satisfaction without added costs of implementation or maintenance. However, further research would be desirable in order to assess the benefits of the program on patients' mental health

KEY WORDS: collaborative care; mental health services; primary health care.

\section{AGRADECIMIENTOS}

Esta investigación es un resumen del trabajo cotidiano de todos los compañeros de los CS y USMC de Andalucía, que para los autores es lo verdaderamente importante. Menciones especiales por su colaboración requieren el Director del centro de San Andrés Torcal el Dr. Francisco Ruiz Solanes, todo su equipo de admisión encabezado por $M^{a}$ Antonia Vega por el trabajo de recogida de información y Carmen Vela del departamento del farmacia del distrito Málaga por elaborar los datos de prescripción del centro de salud.

\section{Introducción}

En el Servicio Andaluz de Salud (SAS) en 1999, las unidades de atención a la salud mental comunitaria (USMC) ${ }^{2}$ realizaron 565.311 consultas; cada facultativo especialista atendió 9.6 revisiones y 1.3 primeras al día, con una demora media de mas de 5 semanas (máximo de 103 con un mínimo de 6 días) (1). En el mismo año, los médicos de familia y pediatras atendieron 49.532 .563 consultas. Este dato combinado con el de primeras visitas a la USMC ponía de manifiesto una importante capacidad de contención, con una derivación del $0.15 \%$ de las demandas de asistencia. Si suponemos que este volumen de consultas lo ha generado el $60 \%$ de la población (que es la que consultaba en un año a su centro de salud en este periodo de tiempo, y aceptamos los resultados de distintas investigaciones sobre prevalencia de trastornos mentales (en torno al 30\%) (2)(3) podemos calcular un índice de derivación a salud mental del 7\% del total de morbilidad psiquiátrica en atención primaria (AP), cifras similares a las del clásico estudio de Goldberg para los médicos generales ingleses (6\%) (4).

\footnotetext{
2 En Andalucía los Servicios de Salud Mental están sectorizados por Áreas (servicios de psiquiatría ubicados habitualmente en el hospital de referencia) de las que dependen las USMC y otros servicios de $3^{\circ}$ nivel (USMI, hospital de día, unidad de agudos, comunidad terapéutica, unidad de rehabilitación). Cada USMC se relaciona con un numero geográficamente determinado de centros de atención primaria.
} 
ORIGINALES Y REVISIONES

Es evidente que estas cifras no mostraban necesariamente un panorama deseable ya que la contención de AP es probable que no fuera tal, sino falta de capacidad para identificar y diagnosticar o escasa accesibilidad de las USMC. Pero alertaban sobre un problema real: las dificultades de los dispositivos asistenciales para satisfacer una demanda potencial que en ese momento o era implícita, o estaba medicalizada, o simplemente era ignorada o pospuesta. La atención a ese $30 \%$ posible en AP (14.859.768 consultas) consumiría el mayor tiempo de dedicación de los médicos de familia en el caso de utilizar solamente como técnica de diagnóstico y tratamiento una entrevista de 15 minutos, también es probable que las derivaciones de la patología detectada saturasen los servicios especializados.

A esto habría que sumar todas las previsiones sobre incremento esperado de la llegada de patología mental a los servicios de salud debido a los múltiples factores ampliamente analizados en otra publicación (5).

Parecía, pues, claro que el problema de la satisfacción de esta demanda potencial era complejo y que un simple aumento de los recursos humanos en cualquier nivel (probablemente necesario) no lo resolvería. Estaba en cuestión el modelo de servicios y su vinculación con otros recursos de contención del sufrimiento mental.

Por estos motivos en el año 2001 la Consejería de Salud de Andalucía se planteó la revisión del modelo de atención a las personas con ansiedad, depresión o somatizaciones (la mal denominada "psiquiatría menor") utilizando un instrumento innovador de planificación centrado en el paciente: la gestión por procesos integrados (6-8).

En ese momento, se podría señalar que estas patologías se presentaban de forma "confusa" o poco discriminada como síndrome ansioso depresivo, sin que fueran identificables, en la mayor parte de los casos, las enfermedades definidas en las clasificaciones internacionales. La oferta de servicios también era "borrosa", salvo el recurso a la medicación, y la demora en la atención especializada dificultaba la continuidad del tratamiento y el acceso a los servicios. La coordinación entre dispositivos y niveles asistenciales era escasa y, en el mejor de los casos, funcionaba como un modelo de derivación donde el primer nivel cumplía la misión de ser filtro para el segundo en la transferencia de pacientes. Si consideramos la comunicación intraequipo (de AP, de la USMC, de urgencias) en torno a pacientes, como un instrumento básico de contención multiprofesional (9), tampoco se contaba con medidas organizativas que aseguraran su existencia y desarrollo.

La propuesta del Proceso de Ansiedad, Depresión, Somatizaciones (PAIADS) (10) era innovadora porque descansaba sobre un modelo de colaboración entre AP y atención especializada (AE). En este modelo están diferenciados los espacios de trabajo de cada nivel con criterios consensuados de transferencia de casos, pero además de ello hay un espacio de trabajo común, cuyo resultado esperado para AP es el aumento de la capacidad real de contención no medicalizadora y para la AE 
la despsiquiatrización y despsicologización del sufrimiento mental en un enfoque biopsicosocial. La comunicación fluida entre niveles genera modelos particulares (no generales) donde se construye la capacidad de contención de la angustia (de pacientes y de profesionales) y cuya definición última es producto de las características específicas de los profesionales de ambos niveles.

En el espacio de AP se buscaba un aumento de la capacidad de reconocer, contener, tratar y referir estas demandas. El reto básico era incrementar la voluntad para asumir como propio de su trabajo la atención a esta morbilidad. El incremento de la oferta de servicios (técnicas de escucha y apoyo psicológico no reglado, grupos psicoeducativos, uso de recursos sociales, desmedicalización, normalización e higienización), las medidas organizativas (responsables de programa, gestión de citas y demora desde AP, objetivos pactados) y la formación fueron los instrumentos propuestos.

Del espacio de AE (USMC y urgencias) se esperaba el refuerzo de la coordinación intraequipo en torno a los pacientes (planes cerrados de tratamientos, reuniones de primeras, evaluación), pero fundamentalmente un reconocimiento de la AP como agente activo en el dispositivo de salud mental. Reconocer al "otro" en este caso significaba un esfuerzo por mejorar la comunicación formal e informal (referentes conocidos para cada centro de salud (CS), remisión sistemática de informes de calidad, fluidez en la resolución de imprevistos, desburocratización) y entender la formación no como el requisito para la colaboración sino como un producto bidireccional del trabajo conjunto.

Sin duda lo más innovador del modelo (y el motor de su desarrollo e implantación) era la creación de un espacio específico de colaboración entre niveles en los CS a través del desplazamiento periódico de los profesionales de AE para ver pacientes conjuntamente (supervisión, coterapia, interconsulta (10)) y hablar de ellos en las sesiones formativas posteriores (Tabla 1).

El documento del Proceso Asistencial Integrado Ansiedad, Depresión, Somatizaciones se finalizó el 20 de junio del 2001 y se publicó y presentó oficialmente en Noviembre del mismo año. Su aplicación a la estructura de servicios se realizó por procedimientos administrativos similares a todos los procesos asistenciales en la estructura jerárquica del SAS, incluyéndose en los contratos-programa de las instituciones a partir del año 2002.

Han pasado 10 años y, aunque han aumentado los recursos (especialmente de SM), siguiendo todas las previsiones la demanda general y específica de salud mental se ha incrementado considerablemente (en Andalucía de 6.8 a 9.2 visitas a AP por persona y año). Es hora, pues, de evaluar el grado de implantación del Proceso en el SAS y sus efectos desde su formulación. En concreto esta investigación pretende evaluar y reflexionar sobre: 
ORIGINALES Y REVISIONES

Tabla 1

Componentes iniciales y añadidos en su implantación, del modelo colaborativo definido en el Proceso Asistencial Integrado Ansiedad, Depresión, Somatizaciones

\begin{tabular}{|c|c|}
\hline stos & $\begin{array}{l}\text { Componentes añadidos en la implantación } \\
\text { (no generalizados) }\end{array}$ \\
\hline $\begin{array}{l}\text { - Gestión de } 1^{\text {a }} \text { consultas especializadas de Salud Mental } \\
\text { desde los Centros de Atención Primaria } \\
\text { - Desplazamiento periódico de FEAs de Salud Mental a } \\
\text { los Centros de Salud } \\
\text { - Consultas conjuntas (interconsulta, supervisión, coterapia) } \\
\text { - Reglamentación de la comunicación formal (informes) } \\
\text { - Responsables del Proceso en Centros de Salud y USMC } \\
\text { - Formación continuada en los Centros de Salud } \\
\text { - Protocolos conjuntos } \\
\text { - Inclusión de objetivos específicos en contratos de gestión }\end{array}$ & $\begin{array}{l}\text { - Filtro de derivaciones } \\
\text { - Historias clínicas compartidas (aplicación informática) } \\
\text { - Reuniones periódicas interinstitucionales de gestión y } \\
\text { evaluación del proceso } \\
\text { - Facilitadores de la comunicación informal } \\
\text { - Rotaciones de profesionales de atención Primaria por } \\
\text { las USMC } \\
\text { - Formación conjunta de residentes (PIR, MIR de medicina } \\
\text { de familia y psiquiatría) } \\
\text { - Rotaciones PIR y MIR de psiquiatría por Centros de } \\
\text { Salud } \\
\text { - Proyectos conjuntos de investigación }\end{array}$ \\
\hline
\end{tabular}

1. La dinámica de implantación y los efectos de dicho modelo en los profesionales de ambos niveles y en los propios pacientes.

2. El grado de penetración del PAIADS en la estructura administrativa, su aplicación real en el conjunto de los servicios, sus modalidades de desarrollo y sus resultados iniciales.

\section{Material y Método}

Para el objetivo $\mathrm{n}^{\circ} 1$ se decidió hacer el análisis en profundidad de un centro de salud urbano ${ }^{3}$. La selección no se hizo con ninguna pretensión de representatividad, sino meramente, por la facilidad que suponía la existencia de registros para evaluar retrospectivamente la experiencia y la voluntad de colaboración de sus profesionales. El caso no tiene carácter piloto, la implantación del PAIADS se inició en varios centros a la vez, incluso había otros que llevaban años de experiencias previas con modelos de colaboración. Es un CS en un barrio consolidado de clase trabajadora fuertemente golpeado por la crisis y con una alta tasa de inmigración.

\footnotetext{
3 Centro de salud San Andrés-Torcal de Málaga.
} 
Atiende a 20.226 personas y cuenta con una plantilla de 11 médicos de familia, 3 pediatras, 11 enfermeras y 6 administrativos, trabajador social y matrona compartidos. Están en condiciones medias de presión asistencial y tamaño de cupos (entre 40-50 consultas diarias de medicina de familia con cupos entre 1500-1600 personas), que han permanecido estables durante estos 10 años y con un nivel alto de motivación y formación de los profesionales ${ }^{4}$. La implantación del PAIADS comenzó en el año 2002. La USMC de referencia era de nueva creación y con una coordinadora y equipo especialmente motivados. Se analizó el modelo organizativo implantado, su evolución en el tiempo, las dificultades y logros percibidos por los profesionales y los resultados medidos por la evolución de las derivaciones realizadas a la USMC (número, características clínicas, calidad) y la prescripción de psicofármacos en el periodo 2007-10. Se combinaron métodos cualitativos (análisis de actas de reuniones y entrevistas en profundidad con actores clave) y cuantitativos (análisis de registros del programa, base de datos de farmacia).

El objetivo $\mathrm{n}^{\circ} 2$ requería de un instrumento que proporcionase información que en su mayor parte no se recogía en los registros de actividad de las unidades clínicas. La única aproximación posible fue la realización de un cuestionario al responsable del programa de Salud Mental del CS (o en su defecto al director) y al coordinador de la USMC. La recogida de datos se realizó desde la Escuela Andaluza de Salud Pública, con la dirección del Plan de Salud Mental de Andalucía (PISMA). Tras el envío de una carta de presentación del proyecto y la remisión por correo electrónico corporativo del cuestionario, un encuestador independiente realizó por teléfono la encuesta a la totalidad de los centros. La información se completó con otras bases de datos oficiales. El periodo de evaluación fue de Noviembre 2008-Mayo 2009. El análisis se realizó usando el paquete SPSS con descriptiva de frecuencias y análisis bivariante.

\section{Resultados}

Objetivo $\mathbf{n}^{\mathbf{0}}$ 1: El centro de salud estudiado escogió este proceso asistencial para ensayar su implantación desde el año 2002, la elección fue conjunta con el resto de los centros dependientes de la misma USMC.

Como primera medida con el objetivo de fortalecer los vínculos profesionales y disminuir la demora existente (más de 90 días) la USMC decidió desplazar a una

\footnotetext{
4 Un médico había participado en el grupo de elaboración del PAIADS, tres médicos tenían formación especifica en psicoterapia y 8 médicos y 2 enfermeras en entrevista clínica. El centro era docente para post y pregraduados.
} 
ORIGINALES Y REVISIONES

psiquiatra y a un enfermero de salud mental a todos los centros de salud durante 1 mes para sesiones de formación y revisar las derivaciones. Esta acción generó resistencias y enfrentamientos entre niveles asistenciales que ya han sido objeto de análisis en otra investigación, (11) pero propició el primer encuentro de instituciones para discutir la estrategia de puesta en marcha del trabajo conjunto. En esta reunión se sentaron las bases del modelo de colaboración que contaba, en ese momento, con los siguientes elementos:

- Un sistemas de visitas quincenales de FEAs de la USMC a cada CS para atender pacientes conjuntamente y organizar sesiones de formación

- Nombramiento de médico de familia y FEA de la USMC responsables del PAIADS en cada CS. Ambos serían los interlocutores y facilitadores de la comunicación entre instituciones, el médico de familia organizaría las citas conjuntas en el CS y el FEA se desplazaría periódicamente y sería siempre el mismo para cada CS

- Establecimiento de un cupo de primeras visitas para cada CS (en función del histórico y la población atendida) que serían gestionadas desde Atención Primaria. A partir de ese momento la lista de espera de primeras visitas sería responsabilidad de cada CS

- Criterios mínimos medico-administrativos para aceptar una derivación como valida: datos de filiación del paciente y del medico derivante, motivo de consulta, problemas de salud activos, medicación que este tomando actualmente, quien hace la demanda, situación laboral y familiar e impresión diagnostica. Los tres primeros obligatorios y de los 6 restantes al menos 4 .

- Reuniones periódicas de evaluación entre las instituciones de ambos niveles asistenciales

A partir de ese momento las dinámicas de implantación del PAIADS en cada centro fueron distintas. En el caso que estudiamos, los hechos característicos fueron las tareas que asumió el responsable del programa en 4 campos específicos.

El primero fue la comunicación bidireccional entre instituciones y profesionales comprobada fuente de malestar y conflictos. Medidas tan sencillas como la presencia de listados de teléfonos de todos los FEA, disponibles en cada consulta, y la pronta resolución de quejas en torno a la asistencia de pacientes, especialmente las urgencias, crearon un ambiente de trabajo facilitador de la colaboración y no del enfrentamiento. El segundo fue la organización de la consulta conjunta (y la formación posterior), estableciendo un sistema de citación de pacientes y, sobre todo, de acogida del facultativo especialista que se desplaza, para evitar que hubiese contratiempos (no hay consulta disponible, no está el paciente o el médico de familia que lo cita, no hay nadie a quien preguntar) que generase en el FEA la sensación de falta de reconocimiento al esfuerzo realizado.

El tercer aspecto fue la función de filtro que estableció el CS. Inicialmente esta función tenía un doble sentido: revisar el cumplimiento del acuerdo sobre los 
datos imprescindibles que debían contener las derivaciones y vigilar la demora de primeras consultas. Para ello el servicio de admisión no daba las citas directamente a los pacientes derivados, sino que almacenaba las peticiones que eran revisadas por el responsable una vez a la semana. A lo largo de los años el sistema fue haciéndose más complejo a medida que los acuerdos con la USMC progresaban y se hacían mas nítidos los criterios de transferencia de pacientes (qué considerar primera visita, protocolo clínico consensuado de derivación de pacientes). Así por ejemplo dejó de considerarse primera visita los pacientes que pedían cambio de terapeuta en la USMC, las recaídas dentro del año posterior al alta, los adelantos de citas en pacientes con exacerbación de sus síntomas, los traslados de pacientes desde otras USMCs, las urgencias vistas por psiquiatras de guardia y remitidas para seguimiento. Todas esas peticiones fueron trasladadas para su gestión al servicio de admisión de la USMC.

En la tabla $\mathrm{n}^{\mathrm{O}} 2$ aparecen los resultados de la aplicación de los 4 filtros a las derivaciones del periodo noviembre 2009-noviembre 2010 (¿es primera visita? ¿cumple criterios médico-administrativos? ¿pertenece al PAIADS? ¿cumple criterios clínicos?). Puede comprobarse que de las 311 derivaciones 21 no correspondían a solicitud de $1^{\mathrm{a}}$ visita (6.8\%), si a esto le sumamos las $14(4.8 \%)$ que fueron devueltas a los médicos derivantes por falta de datos, comprobamos la eficacia del filtro en reducir, sin coste, el número de derivaciones y aumentar su calidad (11.6\%). En la misma tabla encontramos que del total de derivaciones $68.1 \%$ corresponden al PAIADS confirmando que este grupo de patologías suponen la carga mayor del trabajo ambulatorio de la USMC (el 31.9\% restante se distribuye en $34.6 \%$ psicosis, $48.1 \%$ patología de niños y adolescentes, $17.3 \%$ trastornos de la personalidad).

Tabla 2

Análisis del total del numero de derivaciones del CS a la USMC en el periodo Nov 2009- Nov 2010

\begin{tabular}{|l|c|c|c|c|c|c|c|c|}
\hline \multirow{2}{*}{$\begin{array}{l}\text { Total } \\
\text { derivaciones }\end{array}$} & \multicolumn{2}{|l|}{$\begin{array}{l}\text { Corresponden a } \\
\mathbf{1}^{\mathbf{a}} \text { visita }\end{array}$} & \multicolumn{2}{l|}{$\begin{array}{l}\text { Cumplen criterios } \\
\text { administrativos }\end{array}$} & \multicolumn{2}{l|}{$\begin{array}{l}\text { Pertenecen al } \\
\text { PAIADS }\end{array}$} & \multicolumn{2}{l|}{$\begin{array}{l}\text { Cumplen criterios } \\
\text { clínicos derivación }\end{array}$} \\
\cline { 2 - 9 } & $\mathrm{Si}(\%)$ & $\mathrm{No}(\%)$ & $\mathrm{Si}(\%)$ & $\mathrm{No}(\%)$ & $\mathrm{Si}(\%)$ & $\mathrm{No}(\%)$ & $\mathrm{Si}(\%)$ & $\mathrm{No}(\%)$ \\
\hline 311 & $290(93.2)$ & $21(6.8)$ & $276(95.2)$ & $14(4.8)$ & $188(68.1)$ & $87(31.9)$ & $113(60.1)$ & $75(39.9)$ \\
\hline
\end{tabular}

Es de resaltar que sólo tras 7 años de trabajo conjunto se decide hacer un protocolo clínico consensuado de derivación (en el año 2009), lo que supuso una nueva tarea al filtro semanal de las derivaciones. El sentido de esta, no era devolver la derivación que no se ajustaba a protocolo, sino identificar las áreas en las que los médicos de familia se sentían menos cómodos tratando a sus pacientes a fin de articular medidas que pudiesen mejorar su capacidad de contención. Com- 
probamos que más del $60 \%$ de estas se ajustan a lo pactado y que las causas de incumplimiento más frecuentes (tabla $\mathrm{n}^{\circ} 3$ ) fueron los trastornos adaptativos sin intento de tratamiento $(20.7 \%)$ y las derivaciones inducidas por otros especialistas $(4.8 \%)$ que sugieren medidas de mejora de naturaleza completamente distinta. La tabla muestra que los médicos de familia tratan o inician tratamiento de la mayor parte de la patología del PAIADS que detectan, suponiendo el mayor volumen de derivación los trastornos con ánimo depresivo.

Tabla 3

Características clínicas de la patología derivada del CS a la USMC del PAIADS en el periodo

Nov 2009- Nov 2010

\begin{tabular}{|c|c|c|}
\hline Características clínicas de la derivación & $\begin{array}{l}\text { Cumple criterios } \\
\text { pactados de derivación }\end{array}$ & $\mathrm{N}(\%)$ \\
\hline \multicolumn{2}{|l|}{ Trastornos con animo depresivo predominante } & 88(46.8) \\
\hline Depresión sin respuesta al tratamiento inicial & SI & $32(17)$ \\
\hline Depresión severa (intento de autolisis, alto riesgo de suicidio, grave) & SI & 18(9.6) \\
\hline Depresión recurrente & SI & $17(9)$ \\
\hline Depresión para psicoterapia (solicitud o trastorno de la personalidad asociado) & SI & $7(3.7)$ \\
\hline Depresión moderada no tratada & NO & $8(4.3)$ \\
\hline Distimia sin respuesta al tratamiento & SI & $3(1.6)$ \\
\hline Distimia sin intento de tratamiento & NO & $3(1.6)$ \\
\hline \multicolumn{2}{|l|}{ Trastornos con animo ansioso predominante } & 21(11.2) \\
\hline Agorafobia & SI & $5(2.7)$ \\
\hline Ansiedad para psicoterapia & SI & $2(1.1)$ \\
\hline Ansiedad por falta de control de impulsos & SI & $1(0.5)$ \\
\hline Estrés postraumático & SI & $1(0.5)$ \\
\hline Ansiedad en paciente con TOC & SI & $1(0.5)$ \\
\hline Ansiedad en paciente con trastorno de la personalidad & SI & $1(0.5)$ \\
\hline Crisis de pánico sin respuesta al tratamiento & SI & $1(0.5)$ \\
\hline Ansiedad sin respuesta el tratamiento & SI & $4(2.1)$ \\
\hline Cuadro ansioso sin historiar & NO & $4(2.1)$ \\
\hline Fobia simple no tratada & NO & $1(0.5)$ \\
\hline \multicolumn{2}{|l|}{ Somatizaciones } & 3(1.6) \\
\hline Cuadro somatomorfo sin respuesta al tratamiento & SI & $3(1.6)$ \\
\hline \multicolumn{2}{|l|}{ Trastornos adaptativos } & 62(33) \\
\hline Duelo prolongado sin respuesta al tratamiento & SI & $1(0.5)$ \\
\hline Duelo de alto riesgo & SI & $4(2.1)$ \\
\hline Trastorno adaptativo sin respuesta al tratamiento & SI & $17(9)$ \\
\hline Trastorno adaptativo desea apoyo especializado & SI & $1(0.5)$ \\
\hline Trastorno adaptativo no tratado & NO & $39(20.7)$ \\
\hline \multicolumn{2}{|l|}{ Patología dual } & $\mathbf{1}(\mathbf{0 . 5})$ \\
\hline Ansiedad grave con riesgo de malos tratos en paciente toxicómano & SI & $1(0.5)$ \\
\hline \multicolumn{2}{|l|}{ Problemas administrativos } & 13(6.9) \\
\hline Derivaciones inducidas por otros especialistas & NO & $9(4.8)$ \\
\hline Informes para inspección & SI & $2(1.1)$ \\
\hline Petición de cambio de terapeuta & NO & $1(0.5)$ \\
\hline Perdidas de $\operatorname{citas}^{\mathrm{a}}$ & SI & $1(0.5)$ \\
\hline TOTAL & & $188(100)$ \\
\hline
\end{tabular}

a El paciente no acude a la cita programada y hay que gestionarle una nueva 
Otra de las finalidades del filtro es vigilar la demora y en función de ella priorizar las derivaciones semanales según la gravedad del cuadro. En el grafico $\mathrm{n}^{\circ} 1$ vemos por trimestres la evolución de la demora en días en los últimos 4 años comprobando una tendencia descendente, que se estabiliza para los últimos dos años, asociada al número de derivaciones realizadas.

Gráfico ${ }^{\circ} 1$ :

Evolución de la demora de $1^{a}$ consultas a la USMC por trimestres en el periodo 2007-2010

\begin{tabular}{|l|l|l|l|l|}
\hline Trimestre & 2007 & 2008 & 2009 & 2010 \\
\hline $1^{\mathbf{o}}$ Trimestre & 12 & 17 & 17 & $\mathbf{2 1}$ \\
\hline $2^{\mathbf{o}}$ Trimestre & 11 & 14 & 9 & 10 \\
\hline $3^{\mathbf{0}}$ Trimestre & $\mathbf{1 8}$ & $\mathbf{4 4}$ & $\mathbf{1 2}$ & $\mathbf{1 5}$ \\
\hline $4^{\mathbf{0}}$ Trimestre & $\mathbf{3 2}$ & $\mathbf{3 5}$ & $\mathbf{1 1}$ & $\mathbf{2 3}$ \\
\hline
\end{tabular}
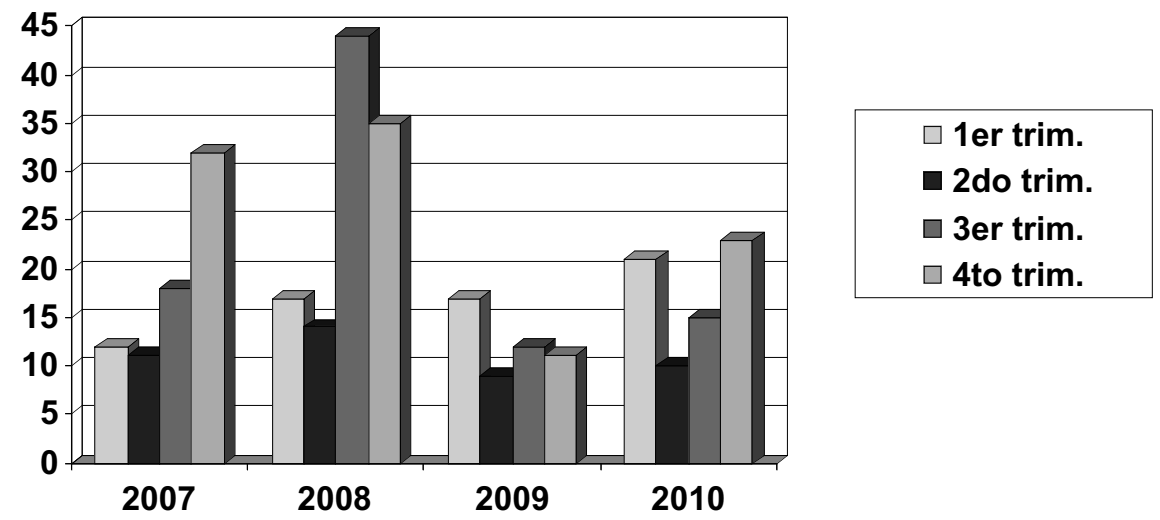

De las entrevistas con los médicos de familia del centro se deduce que nunca la función de filtro se vivió como una imposición, sino más bien una ayuda para su trabajo.

El último aspecto fue la inclusión de los objetivos del programa de salud mental en el contrato de gestión del CS. Desde el 2003 el PAIADS se había incluido como objetivo específico del centro, cuya única norma de calidad era el porcentaje de cumplimiento de los criterios médico- administrativos citados más arriba. Esta norma se cumplió todos los años superando siempre el 95\% del total. En el 2009 las normas se hicieron más complejas por indicación de los servicios centrales del SAS (tabla 4), llevando el 50\% del peso, el control del numero de derivaciones. Puesto que el cumplimiento de este objetivo suponía el $10 \%$ de la productividad global del centro, se facultó al responsable del programa para hacer un seguimiento estrecho del mismo, siendo su relación con la dirección más estrecha. Como se aprecia en el Grafico 2, los objetivos se cumplieron. 
Tabla 4

Objetivos del contrato de gestión año 2009

- Al menos 6 sesiones de formación conjunta,

- Elaboración de un protocolo consensuado de derivaciones,

- Al menos 15 pacientes en coterapia al año,

- Al menos dos grupos psicoeducativos al año,

- No incrementar el $\mathrm{n}^{\circ}$ de las derivaciones mas del $2 \%$ respecto al año previo

Gráfico $\mathrm{n}^{\circ}$ 2:

Evolución del número de derivaciones a la USMC y de los pacientes vistos conjuntamente en el CS en el periodo 2007-2010

\begin{tabular}{|l|c|c|c|c|}
\hline año & 2007 & 2008 & 2009 & 2010 \\
\hline derivaciones & 399 & 429 & 332 & 269 \\
\hline pacientes vistos conjuntamente & 27 & 26 & 57 & 67 \\
\hline
\end{tabular}

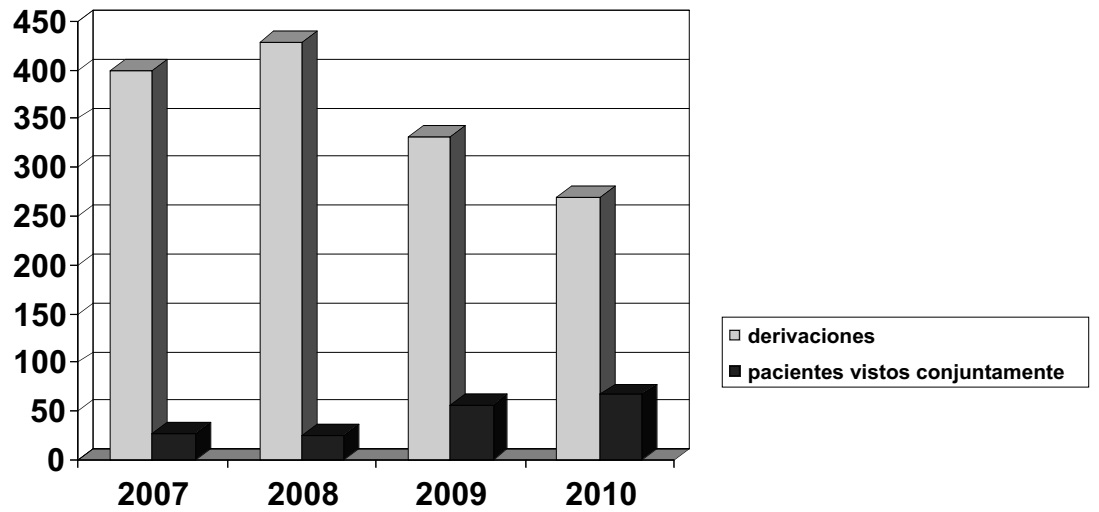

Resulta muy llamativo lo ocurrido en el 2010, donde, aunque la formulación de los objetivos y los indicadores es la misma, el margen de reducción (o no incremento) de las derivaciones era más estrecho ya que se partía de un numero más bajo. Además la repercusión de la crisis económica en el barrio atendido por el CS, significó un incremento de los trastornos adaptativos. Este hecho se puso de manifiesto en la evaluación del $1^{\circ}$ trimestre del 2010 donde las derivaciones a la USMC se habían incrementado un $22 \%$ respecto al mismo periodo del año precedente con alargamiento de la demora. Ante el riesgo de no cumplir los objetivos, la dirección indicó a todos los médicos de familia que los trastornos adaptativos fuesen vistos y tratados en el espacio conjunto del CS. Como se aprecia en el gráfico 2 el número de derivaciones fue controlado y el espacio conjunto más ampliamente utilizado. Este control no implicó aumento de las prescripciones de psicofármacos (disminu- 
ción del 3.4\% de dosis diarias definidas respecto al año anterior), cumpliéndose los objetivos previstos de farmacia para 2010.

En el gráfico $\mathrm{n}^{\circ} 3$ se ve la evolución de la prescripción de psicofármacos en el CS en el periodo 2007-10 comprobándose una disminución del gasto total (15.7\%) y también del coste de la dosis diaria definida (DDD) (19.3\%) con un discreto incremento de las DDD del $2 \%$ (frente al incremento del 22\% del distrito sanitario en su conjunto)

Gráfico $\mathrm{n}^{\mathrm{o}}$ 3:

Evolución de la prescripción de psicofármacos en el Centro de Salud San Andrés Torcal en el periodo 2007-10

\begin{tabular}{|l|c|c|c|c|}
\hline Año & 2007 & 2008 & 2009 & 2010 \\
\hline Importe & 352.675 & 330.129 & 317.106 & 297.186 \\
\hline DDD & 1.154 .075 & 1.184 .806 & 1.218 .332 & 1.176 .949 \\
\hline Coste DDD & 0.31 & 0.28 & 0.26 & 0.25 \\
\hline
\end{tabular}

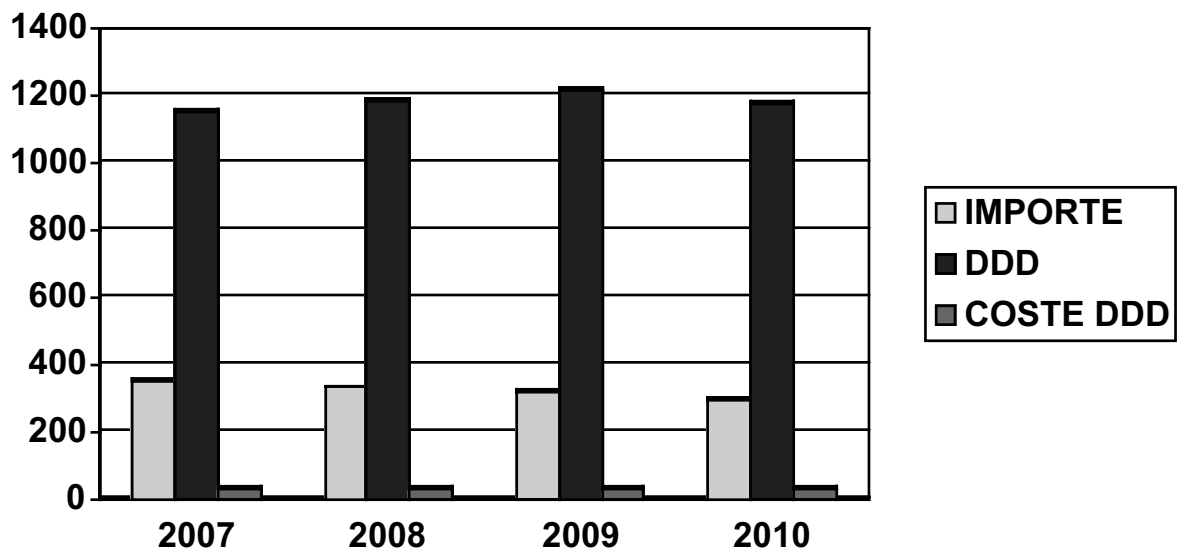

Objetivo no 2: El universo muestral en AP era 380 CS y 76 USMC, la tasa de respuesta fue $68.9 \%$ (n 262) y $78.9 \%$ (n 60) respectivamente. No presenta diferencias estadísticamente significativas según provincia, distritos sanitarios o pertenencia al medio rural o urbano. La causa más frecuente de no respuesta fue no desear participar en el estudio ( $22.4 \%$ en AP y $14.5 \%$ en AE), seguida de imposibilidad de contacto telefónico tras 3 intentos posteriores al envío del cuestionario y la carta de presentación (ausencia de director/coordinador, cambio de dirección y teléfono).

En la Tabla $\mathrm{n}^{\circ} 5$ aparecen las variables recogidas. Como puede observarse solamente el $2.3 \%$ de los centros tienen implantados todos los componentes del proceso, si bien una gran mayoría lleva a cabo alguno de ellos( $88.2 \%$ distribuidos 
Tabla 5

Variables recogidas en el cuestionario telefónico a los Centros

\begin{tabular}{|c|c|c|c|}
\hline Grupo de variables & Variable & Tipo & $\%$ (n) \\
\hline \multirow{6}{*}{$\begin{array}{l}\text { Características asistenciales } \\
\text { - Recursos humanos y materiales } \\
\text { - Población atendida } \\
\text { - Masificación }\end{array}$} & $\%$ cupos de $\mathrm{MF}>1500$ personas $^{\mathrm{a}}$ & Dato & $49.4(2350)$ \\
\hline & $\%$ cupos de Pediatría $>1000$ niños $^{\mathrm{a}}$ & Dato & $64.4(715)$ \\
\hline & CS sin disponibilidad teléfonos SM & Dato & $50.4(127)$ \\
\hline & USMC sin disponibilidad teléfono de CS & Dato & $61.7(37)$ \\
\hline & CS con matrona tiempo completo & Dato & $24(63)$ \\
\hline & CS con Trabajador social tiempo completo & Dato & $38.5(101)$ \\
\hline \multirow[t]{8}{*}{ Aplicación del PAIADS } & $\begin{array}{l}\text { Grado de aplicación global del PAIADS } \\
\text { - Excelente } \\
\text { - Buena } \\
\text { - Alguna } \\
\text { - Solo informes } \\
\text { - Ninguna }\end{array}$ & Dato & $\begin{array}{l}2.3(6) \\
14.1(37) \\
71.8(188) \\
9.2(24) \\
2.7(7) \\
\end{array}$ \\
\hline & $\begin{array}{l}\text { Inclusión de objetivos del PAIADS en el contrato de } \\
\text { gestión de la unidad clínica CS }\end{array}$ & Dato & $11.1(29)$ \\
\hline & $\begin{array}{l}\text { Responsable del PAIADS en USMC y en el CS } \\
\text { - En el CS } \\
\text { - En la USMC }\end{array}$ & Dato & $\begin{array}{l}38.5(101) \\
47.1(97) \\
\end{array}$ \\
\hline & Función de filtro derivaciones en CS & Dato & $14.5(38)$ \\
\hline & Hay desplazamiento periódico de FEA de USMC a los $\mathrm{CS}^{\mathrm{c}}$ & Dato & $45.1(93)$ \\
\hline & Pacientes vistos conjuntamente & Dato & $19.5(51)$ \\
\hline & Formación conjunta & Dato & $40.5(106)$ \\
\hline & Protocolos conjuntos & Dato & $31.3(82)$ \\
\hline \multirow[t]{6}{*}{ Resultados } & $\begin{array}{l}\text { Envío y calidad de los informes (bidireccional) } \\
\text { - Planes Cerrados de Tratamiento a CS } \\
\text { o Calidad Buena /Excelente } \\
\text { - Altas a CS } \\
\text { o Calidad Buena/Excelente } \\
\text { - Calidad derivaciones a SM desde los CS } \\
\text { buena/excelente }\end{array}$ & Opinión & $\begin{array}{l}70.6(185) \\
55.1(102) \\
70.2(184) \\
54.9(101) \\
5.2(3)\end{array}$ \\
\hline & $\begin{array}{l}\text { Tipos de tratamientos realizados en el CS para SM } \\
\text { - Consulta programada SM } \\
\text { - Grupos psicoeducativos }\end{array}$ & Opinión & $\begin{array}{l}59.2(155) \\
22.9(60)\end{array}$ \\
\hline & $\begin{array}{l}\text { Aportaciones del PAIADS } \\
\text { - Poco/ nada según CS } \\
\text { - Poco/nada según USMC }\end{array}$ & & $\begin{array}{l}71.8(188) \\
75.9(45)\end{array}$ \\
\hline & $\begin{array}{l}\text { Satisfacción profesionales } \\
\text { - Buena/excelente en CS } \\
\text { - Buena/excelente en SM } \\
\end{array}$ & Opinión & \begin{tabular}{|l}
$53.4(141)$ \\
$56.7(34)$
\end{tabular} \\
\hline & $\begin{array}{l}\text { Demora en } 1^{\text {a }} \text { consultas a SM } \\
\text { - Menor de } 15 \text { días } \\
\text { - Entre } 15-30 \\
\text { - Mayor de } 30 \text { días }\end{array}$ & Dato & $\begin{array}{l}28.6(44) \\
48.4(74) \\
22.9(35)\end{array}$ \\
\hline & Derivaciones a $\mathrm{SM}>$ media Andalucía ${ }^{\mathrm{d}}$ & Dato & $10.2(21)$ \\
\hline
\end{tabular}

a $\quad$ En Diciembre 2008

b EXCELENTE equivale a: Se hace terapia individual y grupal, existen responsables de SM, FEA acude al CS, pacientes vistos conjuntamente, hay disponibilidad de teléfonos de SM, se realizan sesiones conjuntas, existe un FEA encargado del CS. BUENA: Se hace terapia individual, existe un responsable de SM, FEA acude al CS. ALGUNA APLICACIÓN: Se cumplen algunos de las variables descritas en el caso de Excelente. SOLO INFORMES: la relación entre niveles se limita al envió de informes de derivación y alta. NINGUNA APLICACIÓN: ni siquiera se remiten informes desde la USMC.

c De los que se desplazan el $19.4 \%$ lo hace semanalmente, el $23.5 \%$ quincenal y $57.1 \%$ mensual

d La media de Andalucía es 1.33/1000 habitantes/mes 
en $2.3 \%$ excelente, $14.1 \%$ buena y $71.8 \%$ alguna aplicación), siendo los más generalizados la existencia de responsable del programa (38.5\% en CS y $47.1 \%$ en SM) y el desplazamiento periódico de especialistas de SM a los CS (45.1\%), aunque solamente el $19.5 \%$ ven pacientes conjuntamente con los médicos de familia, dedicándose más a actividades de formación (40.5\%) y realización de protocolos (31.3\%). La función de filtro de las derivaciones a SM existe en el $14.5 \%$ de los CS. El grado de implantación del PAIADS esta asociado estadísticamente con la provincia, el distrito, la inclusión de objetivos de Salud Mental en el contrato de gestión y la satisfacción de los profesionales de atención primaria. No se relaciona con la masificación ${ }^{5}$, la USMC a la que derivan pacientes, la existencia de matrona o trabajador a tiempo completo, la satisfacción de los profesionales de las USMC, el tiempo de demora ${ }^{6} \mathrm{y}$ la tasa de derivaciones.

En la formulación inicial del proceso se incluían una serie de requisitos previos (100\% de cumplimiento) para su puesta en marcha relativos a los recursos humanos $^{7}$, materiales ${ }^{8}$ y organizativos ${ }^{9}$ necesarios, que en el momento de la evaluación se han cumplido sólo parcialmente. Cerca del 50\% de los cupos de medicina de familia y más del $60 \%$ de los de pediatría superan las cifras de pacientes deseables pactadas con sindicatos y sociedades científicas. Mas de la mitad de los CS y las USMC no disponen de fácil comunicación telefónica y en el momento de la evaluación no se había iniciado la aplicación informática de la historia clínica unificada en las USMC. Un porcentaje pequeño de los CS cuentan con matrona y trabajador social a tiempo completo ( 24 y $38.5 \%$ respectivamente).

Solamente el $11 \%$ de los CS han incluido en sus contratos de gestión clínica objetivos del PAIADS existiendo diferencias estadísticamente significativas geográficas y por distritos. Esta variable se asocia con casi todas las de implantación del proceso y sus resultados (desplazamiento periódico de los facultativos especialistas de área (FEA) y su frecuencia, pacientes vistos conjuntamente, existencia de filtro de derivaciones, número sesiones conjuntas, satisfacción de los profesionales, tiempo de demora, realización de grupos psicoeducativos en CS y percepción de aportaciones positivas del PAIADS a los servicios sanitarios).

Lo habitual es la remisión de informes de planes cerrados de tratamiento y altas desde la USMC a los CS (más del 70\%) aunque la calidad de los mismos a juicio de los médicos de atención primaria tiene un amplio margen de mejora (45\%

\footnotetext{
5 La masificación esta medida por las cargas de trabajo (pacientes asignados corregidos por edad) y no por la frecuentación real.

6 Solamente la aplicación excelente reduce el tiempo de demora

7 Menos de 1500 personas por cupo de medicina de familia (Sociedad Andaluza de Medicina Familiar y Comunitaria), menos de 1000 niños por cupo de pediatría (Sociedad Andaluza de Pediatría Extrahospitalaria)

8 Teléfonos disponibles en consulta, equipos informáticos, posibilidad de correo electrónico

9 Inclusión de objetivos de salud mental en contrato de gestión.
} 
ORIGINALES Y REVISIONES

malos o regulares). Por otro lado los facultativos de SM opinan que la calidad de las derivaciones es mala o regular en el $94.8 \%$ de las ocasiones.

Es práctica habitual en los CS la consulta programada para patologías mentales realizada mayoritariamente por médicos de familia (59.2\% vs. $25.6 \%$ de enfermería) y tienen cierta extensión los grupos psicoeducativos (29.9\%) realizados básicamente por enfermería y dedicados preferentemente a relajación, cuidadores y tabaquismo.

A pesar de que la percepción de lo que aporta el PAIADS a la atención a la salud mental es claramente negativa (poco o nada $72 \%$ en CS y $76 \%$ en USMC), la satisfacción de los profesionales por el trabajo conjunto CS-USMC es media alta (buena/excelente $53.4 \%$ en CS y $58.6 \%$ en USMC) en los CS y está relacionada estadísticamente con la aplicación del proceso en conjunto y específicamente con algunos de sus componentes (FEA acude periódicamente al CS y su frecuencia( $35.8 \%$ buena/ excelente de los que no se desplaza vs. 85.7 entre los que se desplaza quincenalmente), FEA responsable del CS, $\mathrm{n}^{\circ}$ de pacientes vistos conjuntamente ( $45 \%$ buena/ excelente de los que no ven pacientes conjuntamente vs. 81.3 entre los que ven mas de 20 en los CS), sesiones conjuntas, protocolos conjuntos, tiempo de demora, y no con el tipo de terapia realizada o el tipo de patología atendida. La satisfacción en los especialistas de SM se relaciona con FEA responsable del CS, FEA acude periódicamente al CS y su frecuencia (39.1\% bue$\mathrm{na} /$ excelente de los que no se desplaza vs. 71.5 entre los que se desplaza) y con la calidad de las derivaciones.

El cuestionario identifica otro aspecto mejorable de la atención: cerca del $23 \%$ de los CS presentan una demora superior al mes para primeras visitas a la USMC. Se asocia a la menor demora la provincia, el distrito, la USMC a la que son derivados los pacientes, el número de pacientes vistos conjuntamente, el número de médicos de primaria con formación en Salud Mental y los protocolos. También existe una clara relación con la satisfacción de los médicos de primaria pero no con la de los FEAs.

Por último la tasa media de derivaciones a las USMC es de 1.33/1000 habitantes al mes, lo que significa que un cupo medio de 1600 personas realiza al año alrededor de 25 derivaciones a los servicios especializados. Aunque el rango llega hasta 5.41/1000 habitantes, solamente el $10.2 \%$ de los CS la superan. El mayor numero de derivaciones esta asociado a la provincia, la masificación (mayor masificación, menor número de derivaciones), a la ausencia de grupos en CS y al no desplazamiento periódico de un FEA a atención primaria. 


\section{Discusión y Conclusiones}

Queremos iniciar la discusión de este artículo con una reflexión sobre los posibles sesgos y utilidades de las fuentes de información utilizadas. En primer lugar el cuestionario tiene un porcentaje de no respuesta del $31.1 \%$ en los CS y de $21.1 \%$ en las USMC siendo la causa más frecuente el no desear participar en el estudio. Aunque la investigación se hizo desde una institución teóricamente fuera de la línea de mando para evitar la sensación de fiscalización de la actividad, no podemos dejar de pensar que no desear participar lleva implícito peores resultados en relación con los indicadores de implantación del PAIADS. Sin embargo no hay diferencias de tasas de respuesta entre provincias, distritos ni medio rural o urbano, por lo que inferimos que el sesgo es geográficamente uniforme hacia ofrecer un panorama probablemente mejor que la realidad de Andalucía. También llamamos la atención sobre la validez de los datos que son opiniones y que pueden incluir un importante sesgo del observador (en general favorable al PAIADS).

Es evidente que el estudio del caso no es generalizable a otros CS. Aunque sea un centro urbano típico, la motivación de los profesionales es probablemente más alta que la media. Si el cuestionario muestra una imagen benévola de Andalucía, la evolución del programa de Salud Mental del centro de salud probablemente sea un óptimo (pero un óptimo real y alcanzable). Pero, para comprender los datos del cuestionario necesitábamos complementar la foto estática y superficial que nos ofrecía, con una visión histórica y en profundidad donde aparecieran descritos los significados de los términos usados y los mecanismos sutiles que transforman organizaciones y culturas enfrentadas en sistemas de cooperación (19).

Los modelos de colaboración en la atención a la salud mental aparecen en el mundo occidental como respuesta a la infradetección e infratratamiento de patología mental en el nivel primario especialmente en la depresión del anciano (12, 13, 14). La motivación inicial del diseño e implantación del PAIADS, como el del resto de procesos asistenciales, estaba relacionada más con un intento de mejora de la calidad total de los servicios usando sistemas de planificación orientados al paciente. En el caso concreto de la salud mental se recogía la tradición basada en los principios de la Psiquiatría Comunitaria y su relación con la Atención Primaria de Salud (15), optándose por un modelo de cooperación, frente al de derivación de pacientes, o los de sustitución de las funciones de un nivel por otro.

La bibliografía refiere experiencias muy diversas, encontrando modelos de sustitución (12), y sistemas similares al PAIADS, que difieren por los componentes organizativos puestos en marcha (sistemas integrados de trabajo (13), case manager (14), registros y bases de datos específicos, auditorias, sistemas de rellamada (16), filtros de derivaciones, protocolos, manuales de procedimientos, formación, reuniones, atención a las urgencias (17) 
ORIGINALES Y REVISIONES

Después de 10 años de trabajo la evaluación tiene que responder a las preguntas ¿es válido el modelo? ¿se han conseguido los objetivos planteados?

Uno de sus propósitos era aumentar la capacidad de detección y de contención no medicalizadora de atención primaria en enfermedades mentales: la hipótesis que queríamos testar es que su aplicación disminuye las derivaciones y consecuentemente la demora también. La información obtenida muestra que la responsabilidad de la demora es compartida con las USMC. Son, pues, dos fenómenos diferentes, el primero depende básicamente de los CS, mientras que el segundo es un resultado de la relación entre ambas instituciones. Que la masificación disminuya el número de derivaciones tira por tierra la hipótesis inicial: la no derivación por infradetección de patología mental por el médico de familia no es un objetivo deseable. El hallazgo interesante es que dos componentes del PAIADS también lo hacen (terapia grupal en el CS y el desplazamiento periódico de los FEAs). Como se ha comentado más arriba la demora es un fenómeno mas complejo, pero también hay componentes manipulables del PAIADS que la disminuyen (numero de pacientes vistos conjuntamente, numero de profesionales formados, existencia de protocolos). Creemos que estos componentes no son sino trazadores de una relación consolidada (que provoca satisfacción en los profesionales del CS) y que su posible implementación sin esa relación, no modificaría la demora de $1^{\text {a }}$ consultas en la USMC.

En el fondo el PAIADS no es más que un conjunto de medidas organizativas sustentadas por un aparato lógico, una forma distinta de articular los recursos existentes que no implica costes adicionales de inversión ni mantenimiento, salvo el esfuerzo de los propios profesionales necesario en cualquier cambio de rutinas. Es por eso que la mejor aproximación al balance coste/beneficio de estas medidas es la satisfacción de los profesionales en relación con el trabajo conjunto. El cuestionario muestra un elevado grado de satisfacción relacionado directamente con la aplicación de los componentes del proceso, especialmente el desplazamiento periódico de los FEAs y su frecuencia o la visita conjunta a pacientes. Sin embargo el merito no es reconocido al PAIADS. Esto nos muestra la importancia de introducir paralelamente los modelos de colaboración a través de vías profesionales (sin olvidar la gran potencia de la inclusión de objetivos del proceso en los contratos de gestión de las unidades clínicas).

Las lecciones mas importantes que sacamos del análisis del caso es que se necesita el tiempo y el conocimiento de las personas, para transformar un modelo basado en el enfrentamiento y la competencia (quien tiene la culpa de...) en uno basado en la cooperación. La propia dinámica clarifica las posiciones: la relación gana en nitidez, se definen los papeles de cada cual y se aprecian tanto los beneficios individuales como los colectivos.Esto facilita la apertura de espacios de sentido de primaria en especializada y viceversa (modificación de los imaginarios 
ORIGINALES Y REVISIONES

colectivos) y la responsabilización de las practicas de cada uno ante el otro (dar cuenta de). Es el único camino para evitar los malos entendidos por proyección de los estereotipos negativos que cada uno tiene de la contraparte, descritos al inicio de esta experiencia (11).

El cumplimiento parcial de los requisitos previos de recursos para la puesta en marcha del PAIADS, a nuestro juicio, significa que, a diferencia de la opinión inicial de los redactores del PAIADS, las dinámicas de relaciones profesionales que se generan son lo suficientemente potentes y satisfactorias para que no existan condiciones previas al ensayo de un modelo de colaboración.

La evolución de la demanda en el 2010 muestra que el modelo tiene capacidad de gestionarla y regularla. Y que esta gestión no implica necesariamente medicalización, ni incremento de la prescripción de psicofármacos en los casos no derivados, sino lo contrario. El margen para reducción de las derivaciones, depende en primera instancia de que los médicos de familia se decidan a tratar (y lo hagan correctamente) los cuadros depresivos y ansiosos con diagnóstico identificable en las clasificaciones internacionales (y tratamientos protocolizables). Para ello es probable que el espacio de visitas conjuntas con los FEAs, les hagan ganar seguridad, también aceptación por parte de sus pacientes y evitar dobles mensajes de estos (trabajan con...) y que la formación y, en última instancia, los protocolos completen el trabajo. Sin embargo vemos que una vez conseguido este objetivo, el caballo de batalla son los trastornos adaptativos, donde la actitud (y el tiempo, y las técnicas) de escucha son el instrumento básico de trabajo. Este es el reto de un modelo que pretende ser contenedor no sólo de las angustias de los pacientes, sino también de las de los propios profesionales.

A nuestro juicio la capacidad de gestión de la demanda se ha generado por dos aspectos: el primero es la autoridad aceptada por el equipo del responsable del programa que se deriva de la inclusión de objetivos de salud mental en el contrato de gestión y la percepción de que la vigilancia de su cumplimiento es una ayuda y no un obstáculo. El segundo es la función de filtro, coincidiendo con los resultados preliminares de Bradford et al (17), para el que la función de filtro es especialmente efectiva en bajar el número de derivaciones, aunque el cómputo global anual no se modifique por el aumento de la capacidad de detección (y derivación) de los médicos generales. En nuestro caso el filtro se asocia a disminución de la demora de primeras consultas y a reducción de las derivaciones.

Si el modelo demuestra su capacidad de contención no medicalizadora, su capacidad de gestión de la demanda y además genera satisfacción en los profesionales, la siguiente pregunta clave es ¿mejora la salud mental de los pacientes?

La revisión sistemática de ensayos clínicos aleatorizados de Huang et al (13) muestra la efectividad del modelo para detectar y tratar la depresión del anciano, aunque Bower et al no logren identificar en su estudio (14) los componentes efecti- 
ORIGINALES Y REVISIONES

vos del modelo. A pesar de ello, lo que la bibliografía deja claro es que la formación interprofesional en colaboración, mejora la capacidad para tratar estas patologías (18). No nos parece especialmente interesante la identificación de componentes organizativos efectivos, porque creemos que estos dependen más de las situaciones particulares de cada sistema sanitario y a nuestro juicio, es más útil el ensayo/ error que la investigación formalizada. En cualquier caso nuestro estudio muestra la importancia de ver pacientes conjuntamente, el desplazamiento periódico de los FEAs, el número de profesionales formados y los protocolos, pero dada la naturaleza transversal de la investigación no sabemos si son causa o consecuencia de un contexto de relación de colaboración. En cualquier caso es una asignatura pendiente la realización de un estudio específico sobre la efectividad de este modelo usando indicadores más generales de salud mental y no sólo de patologías específicas y uso de psicofármacos.

Por último, si esta forma de trabajar demuestra su utilidad, cabría plantearse ¿cómo se extiende el modelo? Por los resultados del estudio parece claro que depende de decisiones propias de los profesionales de primaria, siendo la variable mas potentemente asociada la inclusión de objetivos de salud mental en el contrato de gestión y por tanto la formalización administrativa de esta forma de trabajar.

En definitiva podemos concluir que el PAIADS, como ejemplo de modelo de colaboración de trabajo entre primaria y especializada en salud mental, tiene la capacidad de contención no medicalizadora de la demanda por ansiedad, depresión y somatizaciones en los centros de salud, que esta puede ser gestionada en función de los recursos disponibles y que además genera satisfacción en los profesionales sin costes de implantación o mantenimiento añadidos. Queda pendiente demostrar si mejora la salud mental de los pacientes.

Siglas utilizadas:

AE: atención especializada

AP: atención primaria

CS: centro de salud

DDD: dosis diaria definida

FEA: facultativo especialista de área

PAIADS: proceso asistencial integrado ansiedad, depresión, somatizaciones

PISMA: plan integral de salud mental de Andalucía

SAS: Servicio Andaluz de Salud

SM: salud mental

USMC: unidad de salud mental comunitaria

USMI: unidad de salud mental infantil 
ORIGINALES Y REVISIONES

BIBLIOGRAFÍA:

(1) Memoria Estadística de la Consejería de Salud 1999. Consejería de Salud- Junta de Andalucía. Sevilla 2000

(2) Padierna J.A. et al. La morbilidad psiquiátrica en Atención Primaria Detección y derivación por el medico de familia. Rev. Asoc. Española de Neuropsiquiatría 1988 8: 24-34

(3) Rendueles G; Menéndez MJ; Fernández C et al Psiquiatría ambulatoria de la seguridad social en Asturias. Rev. Asoc. Española de Neuropsiquiatría 1982 2(4): 49-55

(4) D. Goldberg y P. Huxley. "Enfermedad Mental en la Comunidad". Nieva. 1990

(5) Lopez AD, Murray CC. The global burden of disease, 1990-2020. Nat Med 1998; 4(11): $1241-1243$.

(6) Consejería de Salud de la Junta de Andalucía. Guía de diseño y mejora continua de procesos asistenciales. Sevilla. La Consejería; 2001

(7) Revista Calidad Asistencial. Número monográfico sobre gestión de procesos. Junio de 1999, Vol. $14 \mathrm{n}^{\circ} 4$

(8) Revista Calidad Asistencial. Número monográfico sobre modelo europeo de excelencia (EFQM). Abril 2000, Vol. $15 \mathrm{n}^{\circ} 3$

(9) A. Pérez-Sánchez. Practicas Psicoterapéuticas. Ediciones Paidos Barcelona 1996

(10) Consejería de Salud de la Junta de Andalucía. Proceso Asistencial Integrado Ansiedad, Depresión y Somatizaciones. Sevilla. La Consejería; 2002.

(11) Montero M, Lucena E. Liñero J. Grupos de conveniencia o matrimonio a la fuerza. IX Jornadas Internacionales Grupo, Psicoterapia y Psicoanálisis. SEGPA. Gijón 2004

(12) Chew-Graham CA, Lovell K, Roberts C, Baldwin R, Morley M, Burns A, Richards D, Burroughs $\mathrm{H}$. A randomised controlled trial to test the feasibility of a collaborative care model for the management of depression in older people. British Journal of General Practice 2007; 57: 364-370.

(13) Huang Chang-Quan, MD, Dong Bi-Rong, MD, Lu Zhen-Chan, MD, Zhang Yuan, MD, Pu Yu-Sheng, MD, and Liu Qing-Xiu, MD. Collaborative Care Interventions for Depression in the Elderly: A Systematic Review of Randomized Controlled Trials J Investig Med 2009;57: 446-455

(14) Peter Bower, Simon Gilbody, David Richards, Janine Fletcher And Alex Sutton. Collaborative Care For Depression In Primary Care Making Sense Of A Complex Intervention: Systematic Review And meta-Regression. British Journal of Psychiatry (2006), 189, pp484-493

(15) Tizón García JL. Atención Primaria en Salud Mental y Salud Mental en Atención Primaria. Doyma. Barcelona 1992

(16) Richard Byng, Roger Jones, Morven Leese, Blake Hamilton, Paul McCrone and Tom Craig. Exploratory cluster randomised controlled trial of shared care development for long-term mental illness. British Journal of General Practice, 2004, 54, 259-266.

(17) Bradford L. Felker, M.D.Robert F. Barnes, M.D.Diane M. Greenberg, Ph.D.Edmund F. haney, Ph.D.Molly M. Shores, M.D.Linda Gillespie-Gateley, M.S.W., L.I.C.S.W.M. Katherine Buike, M.S.W., L.I.C.S.W.Christine E. Morton, M.R.E. Preliminary Outcomes From an Integrated Mental Health Primary Care Team. Psychiatric Services 55:442-444, 2004

(18) Zwarenstein M, Reeves S, Perrier L. Effectiveness of pre-licensure interprofessional education and post-licensure collaborative interventions. Journal of Interprofessional Care 2005; 19:S148-65.

(19) Felix Castillo. "El profesional en las organizaciones," capítulo 7 de M. Coletti y J. L.Linares: "La intervención sistémica en los servicios sociales ante la familia multiproblemática." Paidos Terapia Familiar, 1997 